\title{
The relationship between chronic diseases and number of sexual partners: an exploratory analysis
}

\author{
Igor Grabovac (ㅇ, , ${ }^{1}$ Lee Smith, ${ }^{2}$ Lin Yang, ${ }^{3}$ Pinar Soysal, ${ }^{4}$ \\ Nicola Veronese, ${ }^{5}$ Ahmet Turan Isik, ${ }^{4}$ Suzanna Forwood, ${ }^{6}$ Sarah Jackson ${ }^{7}$
}

- Additional material is published online only. To view please visit the journal online (http://dx.doi.org/10.1136/ bmjsrh-2019-200352).

'Department of Social and Preventive Medicine, Centre for Public Health, Medical University Vienna, Vienna, Austria

${ }^{2}$ The Cambridge Centre for Sport and Exercise Sciences, Anglia Ruskin University, Cambridge, UK ${ }^{3}$ Alberta Health Services, Calgary, Alberta, Canada

${ }^{4}$ Department of Geriatric Medicine, Bezmialem Vakif University, Istanbul, Turkey ${ }^{5}$ Neuroscience Institute, Aging Branch, National Research Council, Padua, Italy

${ }^{6}$ School of Psychology and Sport Science, Anglia Ruskin University, Cambridge, UK

${ }^{7}$ Department of Behavioural Science and Health, University College London, London, UK

\section{Correspondence to}

Dr Lee Smith, Anglia Ruskin University, Cambridge CB1 1PT, UK; lee.smith@anglia.ac.uk

Received 14 March 2019 Revised 8 December 2019 Accepted 18 December 2019 Published Online First 13 February 2020

Check for updates

(c) Author(s) (or their employer(s)) 2020. No commercial re-use. See rights and permissions. Published by BMJ.

To cite: Grabovac I, Smith L, Yang L, et al. BMJ Sex Reprod Health 2020;46:100-107.

\begin{abstract}
Background We investigated sex-specific associations between lifetime number of sexual partners and several health outcomes in a large sample of older adults in England.

Methods We used cross-sectional data from 2537 men and 3185 women aged $\geq 50$ years participating in the English Longitudinal Study of Ageing. Participants reported the number of sexual partners they had had in their lifetime. Outcomes were self-rated health and selfreported limiting long-standing illness, cancer, coronary heart disease, and stroke. We used logistic regression to analyse associations between lifetime number of sexual partners

\section{Key messages}

- Lifetime numbers of sexual partners is associated with sexually transmitted infection acquisition and the associated health risks which may affect health in later life.

- Using cross-sectional data from a representative sample of older English adults we found that higher number of sexual partners is associated with increased risk of reporting a cancer diagnosis.

- Sexual history may be a relevant clinical indicator for cancer risk in older patients.
\end{abstract} and health outcomes, adjusted for relevant sociodemographic and health-related covariates. Results Having had 10 or more lifetime sexual partners was associated with higher odds of reporting a diagnosis of cancer than having had 0-1 sexual partners in men (OR 1.69, 95\% Cl 1.01 to 2.83 ) and women (OR $1.91,95 \% \mathrm{Cl}$ 1.04 to 3.51$)$, respectively. Women who had 10 or more lifetime sexual partners also had higher odds of reporting a limiting long-standing illness (OR 1.64, 95\% Cl 1.15 to 2.35). No other statistically significant associations were observed

Conclusions A higher lifetime number of sexual partners is associated with increased odds of reported cancer. Longitudinal research is required to establish causality. Understanding the predictive value of lifetime number of sexual partners as a behavioural risk factor may improve clinical assessment of cancer risk in older adults.

\section{BACKGROUND}

There is a large body of literature investigating the relationship between sexual activities, including total number of sexual partners, and risk of developing sexually transmitted infections (STIs). To date, most of this research has focused on adolescents and young adults or the cost-effectiveness of preventative strategies. ${ }^{1}$ Studies have shown that a greater number of sexual partners is associated with greater risk of contracting STIs in adolescents. $^{2}$

STIs can have long-term consequences for health, including greater risk of specific cancers. Rates of human papillomavirus (HPV) infection in sexually active young females have been consistently reported to range from $19 \%$ to $46 \%{ }^{3}$ Nearly all cases of cervical cancer can be attributable to HPV infection. ${ }^{4}$ Moreover, HPV has been found to be associated with cancers of the mouth, penis and anus, ${ }^{4}$ cancers that are most common in older adults. ${ }^{56}$ Other STIs, such as gonorrhoea infection, have been shown to increase the risk of prostate cancer in black men. ${ }^{7}$ The average age for men to be diagnosed with prostate cancer is between 55 and 69 years. ${ }^{8}$ Therefore, STIs may have a long lasting negative impact on adults later in life. Investigations into infection-cancer associations have shown that hepatitis B and hepatitis $\mathrm{C}$ are associated with a much 
higher risk of developing liver cancer, ${ }^{9}$ a common cancer among older adults with a peak rate between the ages of 85 to 89 years. ${ }^{10}$ People living with HIV are also more susceptible to several types of cancers. ${ }^{11}$ Besides cancer, STIs have also been found to be associated with diseases of the cardiovascular system. ${ }^{12}$

It is plausible that a greater number of lifetime sexual partners in older adults increases the risk of contracting an STI over the lifespan and subsequently increases the risk of developing health complications in later life. Given that STIs often go undiagnosed, number of sexual partners could provide a proxy measure of sexual risk behaviour that is more accurately reported (although likely subject to potential underreporting by people with a higher number of partners). Establishing the extent to which number of sexual partners is associated with health problems is important in gauging the potential utility of this measure as an indicator of risk.

Previous studies that have investigated the number of sexual partners and cancer risk have shown mixed findings. In a sample of black men, those reporting 25 or more sexual partners were found to be 2.80 (95\% CI 1.29 to 6.09 ) times more likely to be diagnosed with cancer compared with men with five or fewer partners. ${ }^{13}$ Other research has found similar findings in more diverse samples in relation to anal cancer, prostate cancer and oral cancer. ${ }^{714} 15$ In contrast, a study using a Canadian population found reduced risk of prostate cancer among men with more than 20 sexual partners in their lifetime. ${ }^{16}$ Little research has been carried out examining the role of number of sexual partners as a behavioural risk factor for wider health outcomes in older adults.

To fill these knowledge gaps, the aim of the present study was to investigate the sex-specific associations between the number of lifetime sexual partners and several health outcomes in a large sample of older adults in England. We hypothesised that a greater number of previous sexual partners would be associated with increased risk of unfavourable health outcomes.

\section{METHODS}

\section{Study population}

We used cross-sectional data from the English Longitudinal Study of Ageing (ELSA), a populationrepresentative longitudinal panel study of men and women aged $\geq 50$ years living in England. ${ }^{17}$ The initial ELSA sample was drawn from households with one or more members aged 50 years or older responding to the Health Survey for England (HSE) in 1998, 1999 and 2001. All household members 50 years or older plus partners who were younger than 50 years or who had joined the household since the HSE were invited for interview. Since ELSA began in 2002, data have been collected in biennial waves via computer-assisted personal interview and self-completion questionnaires. The present study uses data from Wave 6 (2012/13) as this is the only wave in which participants have been asked about their number of sexual partners. The Sexual Relationships and Activities Questionnaire (SRA-Q) was administered as a self-completion measure and was returned by 7079 (67\%) participants. We restricted our sample to those who reported their lifetime number of sexual partners and had complete data on all covariates, leaving a final sample for analysis of 5722 men and women. All participants gave fully informed consent to participate in the study, and ethical approval was obtained from the London MultiCentre Research Ethics Committee.

\section{Measures}

Exposure: lifetime number of sexual partners

Number of sexual partners was assessed as part of the SRA-Q, ${ }^{18}$ which participants completed in private and returned in a sealed envelope. The male and female versions of the SRA-Q are available online at http:// www.elsa-project.ac.uk/study-documentation. Participants were asked to indicate the number of sexual partners (vaginal/oral/anal sex) they had had in their lifetime $(0,1,2-4,5-9,10-19,20+)$. Due to the low numbers of participants reporting having had 0 or in excess of 20 partners, we combined these with the proximal categories, resulting in four groups for analysis: $0-1,2-4,5-9$ and $\geq 10$ sexual partners.

\section{Health outcomes}

Self-rated health was assessed using a single item: "Would you say your health is ... poor/fair/good/very good/excellent?". We analysed the proportion of individuals rating their health as fair/poor, as has been done in previous studies. ${ }^{19}{ }^{20}$ Limiting long-standing illness was self-reported in response to two questions: (i) "Do you have any long-standing illness, disability, or infirmity? By long-standing I mean anything that has troubled you over a period of time or that is likely to affect you over a period of time”. If yes, (ii) "Does this illness or disability limit your activities in any way?". Affirmation of a long-standing illness and the reporting of any form of limitation classified the participant as having a limiting long-standing illness. Information about doctor-diagnosed cancer, coronary heart disease (CHD) and stroke (ever in their lifetime) was self-reported.

\section{Covariates}

All potential confounders were selected a priori based on previous literature reporting links between these variables and our exposure and outcomes of interest. Demographic information collected included age, ethnicity (white vs non-white) and partnership status (married/cohabiting, separated/divorced, widowed, or single/never married). Socioeconomic status was based on household non-pension wealth which has been identified as particularly relevant to health outcomes in this age group, ${ }^{21}$ categorised into quintiles across all 
Wave 6 ELSA participants. We also included data on several health-related variables. Participants reported their current smoking status (smoker or non-smoker) and frequency of alcohol intake, categorised as never/ rarely (never to once or twice a year), regularly (once every couple of months to twice a week) or frequently (3 days a week to almost every day). Physical activity was assessed with three items that asked participants how often they took part in vigorous, moderate and low-intensity activities (more than once a week, once a week, 1-3 times a month, hardly ever/never), ${ }^{22}$ and further categorised into three groups, as previously described: ${ }^{23}$ inactive (no moderate/vigorous activity on a weekly basis); moderate activity at least once a week; and vigorous activity at least once a week. Depressive symptoms were assessed using the eight-item Centre of Epidemiological Studies Depression (CES-D) scale, a scale highly validated for use in older adults. ${ }^{24}$ These variables have been shown to be linked with number of sexual partners and differences in perceived health and the diagnoses of interest here. ${ }^{25-33}$

\section{Patient and public involvement}

There was no involvement of patients or the public in the design of any aspect of the present study.

\section{Statistical analysis}

Analyses were performed using IBM SPSS Statistics 22 on complete cases. Data were weighted to correct for sampling probabilities and for differential nonresponse and to calibrate back to the 2011 National Census population distributions for age and sex. The weights accounted for the differential probability of being included in Wave 6 of ELSA and for nonresponse to the SRA-Q. Details can be found at https:// www.ucl.ac.uk/drupal/site_iehc/sites/iehc/files/5050_ elsa_w6_technical_report_v1.pdf ${ }^{34}$

Associations between lifetime number of sexual partners and covariates were assessed using one-way analyses of variance (ANOVAs) for continuous variables and chi-square tests for categorical variables. We used logistic regression to analyse associations between lifetime number of sexual partners and fair/poor selfrated health, limiting long-standing illness, cancer, CHD, and stroke. All models were adjusted for age, partnership status, ethnicity, wealth, smoking status, alcohol intake, physical activity, and depressive symptoms. Separate analyses were carried out on men and women. For each outcome, we report the odds ratio (OR) and 95\% confidence interval (95\% CI) for those who reported having had $2-4,5-9$ and $\geq 10$ sexual partners in their lifetime, relative to those who had had $0-1$ sexual partners. To check whether our categorisation of the number of sexual partners (grouping together the 0 and 1 responses and 10-19 and $\geq 20$ responses) influenced the results, we ran sensitivity analyses with distinct groups for those reporting 0,1 , $10-19$ and $\geq 20$ sexual partners.

\section{RESULTS}

There were 2537 men and 3185 women in the sample. The mean age of participants was 64.25 (SD 9.75) years in men and 65.34 (SD 10.06) years in women. The majority were married or cohabiting $(73.6 \%$ of men, $60.6 \%$ of women), white $(93.7 \%$ of men, $96.0 \%$ of women), non-smokers (85.5\% of men, $86.9 \%$ of women) who drank alcohol regularly or frequently (84.0\% of men, $69.9 \%$ of women) and were moderately or vigorously active at least once a week $(80.2 \%$ of men, $74.8 \%$ of women). Among men, $28.5 \%$ reported having had $0-1$ sexual partners in their lifetime, 29.0\% had had between 2 and 4 partners, 20.2\% had had between 5 and 9 partners, and $22.2 \%$ had had 10 or more partners. Among women, the respective figures were $40.8 \%(0-1), 35.5 \%(2-4), 15.8 \%$ (5-9) and $7.8 \%(\geq 10)$.

Sample characteristics in relation to number of sexual partners are summarised in table 1 . In both men and women, a higher number of sexual partners was associated with younger age, being unmarried, and being in either the highest or lowest quintile of wealth. Those with a higher number of sexual partners were also more likely to report smoking, frequent alcohol intake and engaging in vigorous physical activity on a weekly basis. There was an association between higher number of sexual partners and white ethnicity in women but not in men, and an association between higher number of sexual partners and a greater number of depressive symptoms in men but not in women.

Associations between lifetime number of sexual partners and health outcomes are presented in table 2 . Among women, there was a statistically significant association between number of sexual partners and risk of limiting long-standing illness. Relative to women who reported having had $0-1$ sexual partners, the odds of reporting a limiting long-standing illness were $64 \%$ higher for those who had had between 5 and 9 sexual partners in their lifetime (OR 1.64, 95\% CI 1.15 to $2.05, \mathrm{p}=0.003$ ) and $64 \%$ higher for those who had had 10 or more partners (OR 1.64, 95\% CI 1.15 to $2.35, \mathrm{p}=0.007)$. There was also a statistically significant association between number of sexual partners and cancer risk in both women and men. In women, differences between those reporting 2-4 and 5-9 sexual partners and those reporting $0-1$ sexual partners were not statistically significant $(p>0.13)$, but those who had had 10 or more sexual partners in their lifetime had 91\% higher odds of reporting a diagnosis of cancer than those who had had $0-1$ sexual partners (OR 1.91, 95\% CI 1.04 to $3.51, \mathrm{p}=0.038$ ). In men, odds of cancer were increased by $57 \%$ among those reporting 2-4 lifetime sexual partners (OR 1.57, $95 \% \mathrm{CI} 1.02$ to $2.42, \mathrm{p}=0.039)$ and by $69 \%$ among those reporting $\geq 10$ sexual partners (OR $1.69,95 \%$ CI 1.01 to $2.83, \mathrm{p}=0.047$ ) relative to those who had had $0-1$ partners. The difference between men reporting 5-7 sexual partners and those reporting $0-1$ partners 


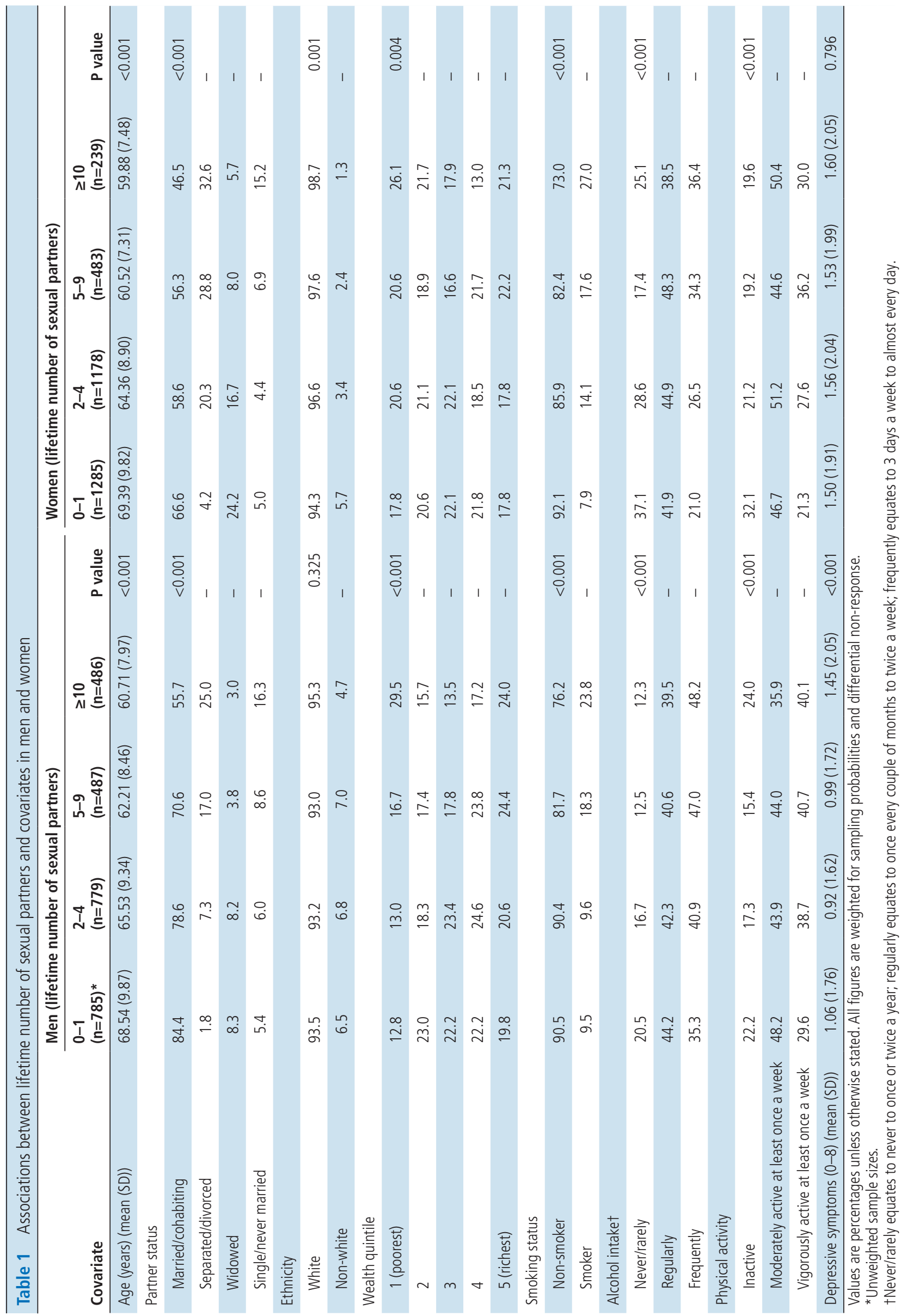




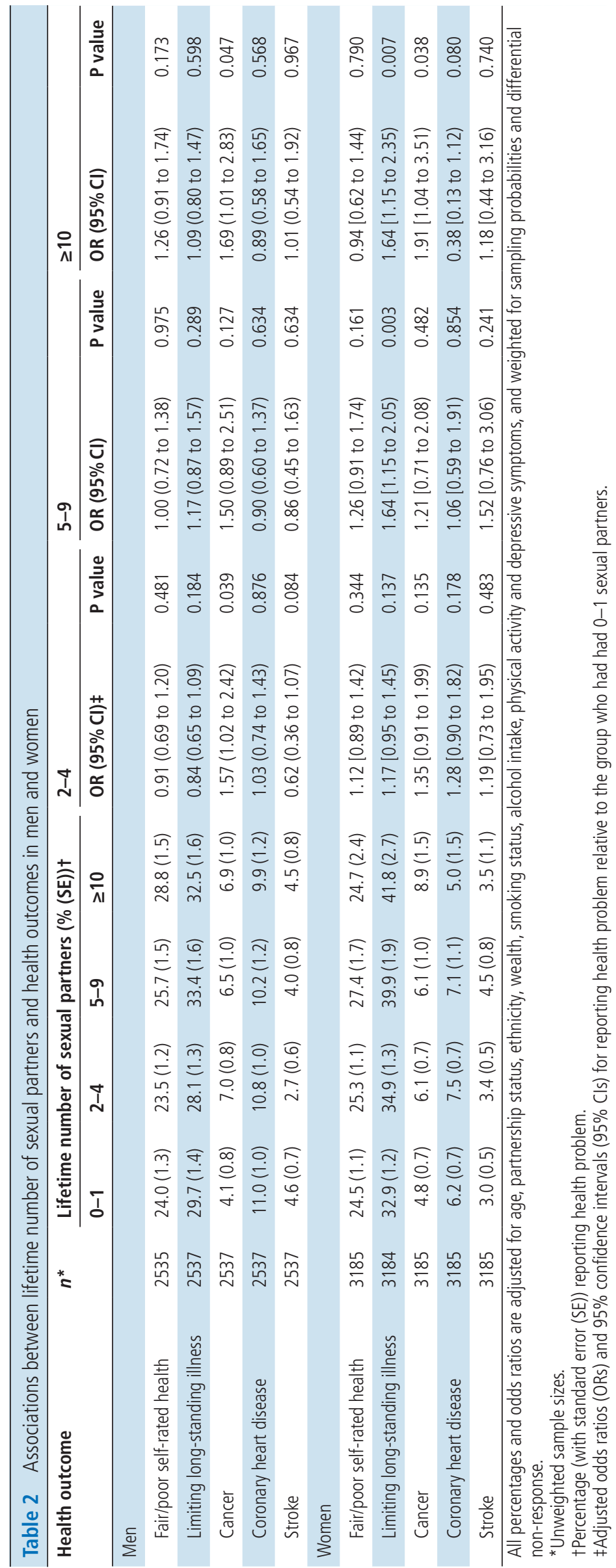


did not reach statistical significance, although the effect size was in the same region as that for the group reporting 2-4 sexual partners (OR 1.50, 95\% CI 0.89 to $2.51, \mathrm{p}=0.127)$. Lifetime number of sexual partners was not statistically significantly associated with self-rated health, CHD or stroke in either sex, or with limiting long-standing illness in men.

Sensitivity analyses in which those reporting 0,1 , $10-19$ and $\geq 20$ lifetime sexual partners were analysed separately revealed no notable differences in the pattern of results (online supplementary table 1).

\section{DISCUSSION}

\section{Summary of findings}

These results provide some evidence that the number of lifetime sexual partners is associated with adverse health outcomes in a sample of older adults in England. In both men and women, a higher number of sexual partners was associated with increased risk of cancer. In women, there was also a statistically significant positive association between number of sexual partners and risk of limiting long-standing illness. We observed no statistically significant association between number of lifetime sexual partners and self-rated health, CHD or stroke in either sex, or with limiting long-standing illness in men.

\section{Comparison with previous studies}

These findings provide some support for the aforementioned hypothesis that a greater number of previous sexual partners is likely to increase the risk of adverse health outcomes in older adults. Perhaps most importantly, the present findings show that a greater number of lifetime sexual partners is associated with increased risk of cancer in older adults. Our findings, using a more proximal outcome, are in line with a large body of literature that suggests that specific STIs may lead to several cancers, such as HPV and cervical, oral, penile and anal cancers, ${ }^{4-6}$ hepatitis $C$ and hepatitis B and liver cancer $^{9}$ as well as gonorrhoea infection and prostate cancer. ${ }^{7}$ This finding supports previous research ${ }^{13-15}$ in suggesting that lifetime number of sexual partners can aid in the identification of those older adults who are at a higher potential risk of cancer. The limited number of specific cancer cases precluded further analysis by cancer type. We speculate the heightened risk of cancer might be driven by those types known to be associated with STIs. Further studies using large samples to elucidate such associations is important in evaluating the potential utility of health practitioners screening older adults for number of lifetime sexual partners when considering risk of specific cancers.

The finding that number of lifetime sexual partners is associated with limiting long-standing illness in women and not men should be noted. This gender difference is interesting, but an explanation is elusive, especially when men have a greater number of lifetime sexual partners than women, as shown in this study, and women are more likely to seek medical screening for STIs ${ }^{35}$ and are thus less likely to experience negative long-term health complications. Further research is required to identify mechanisms that explain this observed association and the divergent pattern between men and women.

While some previous studies have suggested that STIs are associated with diseases of the cardiovascular system, ${ }^{12}$ the present results provide no evidence that the number of lifetime sexual partners is associated with risk of CHD or stroke. It may be that only specific STIs, such as HIV and hepatitis C, are associated with cardiovascular health. ${ }^{12}$ It is possible that these infections are contracted less often than the multiple STIs that can lead to cancer, and thus a precise measure of these exposures is needed to observe a statistically significant association. Moreover, the biological processes between STIs and cardiovascular disease may be weaker than those driving cancer. Further research is required to ascertain the causal mechanisms driving such associations.

\section{Implications and directions for further research}

Our findings indicate a potential utility of lifetime number of sexual partners as a behavioural factor for cancer risk assessment. In our analysed sample, divergent lifestyle profiles have been observed in relation to lifetime number of sexual partners. Those individuals with a greater number of sexual partners were more likely to smoke cigarettes and drink alcohol frequently; behaviours known to be associated with cancer risk. ${ }^{367}$ It is possible that the number of sexual partners one has had captures a combination of likelihood of exposure to STIs and lifestyle profile.

Further research is required to replicate and confirm our findings. We tested a number of models and it is possible that the association between number of sexual partners and cancer was a chance finding. If the same associations were observed, it would be interesting to explore the extent to which the associations are moderated by key sociodemographic (eg, socioeconomic position), health-related (eg, physical activity) and sex-related (eg, sexual orientation) variables. One could also explore the predictive value of lifetime number of sexual partners as a simple question (eg, please indicate the number of sexual partners (vaginal/ oral/anal sex) you have had in your lifetime $(0,1,2-4$, $5-9,10-19,20+)$ embedded in routine clinical assessment for cancer risk.

\section{Strengths and limitations}

Strengths of this study include the large sample and statistical adjustment for a number of important covariates. Moreover, the older age of the sample corresponds to the time of life when our outcomes of interest tend to become more prevalent. However, the findings from the present study must be interpreted in the light of its limitations. First, all data 
were self-reported, which introduces a number of potential biases. For example, the measure of number of sexual partners may have been subject to social desirability bias, although this item was asked in a paper-based questionnaire returned by post rather than in a face-to-face interview, to minimise participant embarrassment and encourage honest responses. Diagnoses of cancer, CHD and stroke may not have accurately been recalled, although previous studies have shown high agreement between selfreported cancer diagnoses and medical record validation in population-based samples. ${ }^{38-40}$ There is also the possibility that self-reports may lack accuracy in older participants with memory problems. Second, the number of sexual partners was assessed on a categorical response scale which asked participants to select the range within which their number of sexual partners fell. This meant we did not have the precise number of sexual partners for most participants (ie, all those who had had more than one sexual partner) and as such it was not possible to model this exposure as a continuous variable, which would have provided more easily interpretable results regarding the increase in risk of cancer associated with each additional sexual partner. Third, no data were available on partner gender (eg, men having sex with men), which may be linked with higher risk of contracting certain STIs. Fourth, the small number of cancer diagnoses meant we lacked statistical power to analyse this outcome broken down by cancer type. It is likely that the association between number of sexual partners and cancer would be stronger for those known to be associated with STIs. Fifth, there was a substantial amount of missing data due to nonresponse to the survey assessing sexual relationships, so these findings cannot be presumed to generalise to the entire population of older adults in England. Further research is required to replicate our analysis and examine whether the same associations are observed in different populations. Moreover, if those participants who had had fewer (or indeed, more) partners were more likely to live to the time of survey then associations between number of sexual partners and health outcomes may have been underestimated. There may be a potential survival bias in the present analyses since the reference group $(0-1)$ was statistically significantly older than people in the $>10$ group. In other words, it is possible that cancer rates were even higher in the group with more sexual partners, but these people had already died. Finally, the analyses were cross-sectional and, as such, it is not possible to determine causality, or even whether the number of sexual partners pre-dated any health diagnoses. It is possible that some people may react to diagnosis of a chronic condition by 'living life to the full', resulting in a rise in their number of lifetime sexual partners. Further research using a prospective design could provide further insight.

\section{CONCLUSIONS}

In this large sample of older adults in England we found that a greater number of previous sexual partners was associated with increased odds of cancer in men and women, and increased odds of limiting long-standing illness in women only. Enquiring about the number of sexual partners a patient has had may be a simple and cost-effective complement to existing cancer screening programmes in identifying those at risk of certain cancers, although further work is required first in order to replicate our findings and establish whether a causal relationship exists.

Contributors IG, LS and SJ conceived the idea, carried out the analyses, interpreted the results, and drafted the manuscript. All authors provided critical revisions and approved the final manuscript prior to submission.

Funding The authors have not declared a specific grant for this research from any funding agency in the public, commercial or not-for-profit sectors.

Competing interests None declared.

Patient consent for publication Not required.

Provenance and peer review Not commissioned; externally peer reviewed.

Data availability statement Data are available in a public, open access repository.

ORCID iD

Igor Grabovac http://orcid.org/0000-0001-9605-1467

\section{REFERENCES}

1 Korenromp EL, Wi T, Resch S, et al. Costing of national STI program implementation for the global STI control strategy for the health sector, 2016-2021. PLoS One 2017;12:e0170773.

2 Stergachis A, Scholes D, Heidrich FE, et al. Selective screening for Chlamydia trachomatis infection in a primary care population of women. Am J Epidemiol 1993;138:143-53.

3 Moscicki ABet al. Risks for incident human papillomavirus infection and low-grade squamous intraepithelial lesion development in young females. JAMA 2001;285:2995-3002.

4 de Martel C, Plummer M, Vignat J, et al. Worldwide burden of cancer attributable to HPV by site, country and HPV type. Int J Cancer 2017;141:664-70.

5 Arya M, Li R, Pegler K, et al. Long-term trends in incidence, survival and mortality of primary penile cancer in England. Cancer Causes Control 2013;24:2169-76.

6 Wilkinson JR, Morris EJA, Downing A, et al. The rising incidence of anal cancer in England 1990-2010: a populationbased study. Colorectal Dis 2014;16:O234-9.

7 Sarma AV, McLaughlin JC, Wallner LP, et al. Sexual behavior, sexually transmitted diseases and prostatitis: the risk of prostate cancer in black men. J Urol 2006;176:1108-13.

8 Hayes JH, Barry MJ. Screening for prostate cancer with the prostate-specific antigen test: a review of current evidence. JAMA 2014;311:1143-9.

9 Perz JF, Armstrong GL, Farrington LA, et al. The contributions of hepatitis $\mathrm{B}$ virus and hepatitis $\mathrm{C}$ virus infections to cirrhosis and primary liver cancer worldwide. J Hepatol 2006;45:52938.

10 Cancer Research UK. Liver cancer incidence statistics, 2018. Available: https://www.cancerresearchuk.org/health- 
professional/cancer-statistics/statistics-by-cancer-type/livercancer/incidence [Accessed 31 Aug 2018].

11 Judd A, Zangerle R, Touloumi G, et al. Comparison of Kaposi sarcoma risk in human immunodeficiency virus-positive adults across 5 continents: a multiregional multicohort study. Clin Infect Dis 2017;65:1316-26.

12 Freiberg MS, Chang C-CH, Skanderson M, et al. The risk of incident coronary heart disease among veterans with and without HIV and hepatitis C. Circulation 2011;4:425-32.

13 Daling JR, Madeleine MM, Johnson LG, et al. Human papillomavirus, smoking, and sexual practices in the etiology of anal cancer. Cancer 2004;101:270-80.

14 Dennis LK, Dawson DV. Meta-analysis of measures of sexual activity and prostate cancer. Epidemiology 2002;13:72-9.

15 Schwartz SM, Daling JR, Doody DR, et al. Oral cancer risk in relation to sexual history and evidence of human papillomavirus infection. J Natl Cancer Inst 1998;90:1626-36.

16 Spence AR, Rousseau M-C, Parent Marie-Élise. Sexual partners, sexually transmitted infections, and prostate cancer risk. Cancer Epidemiol 2014;38:700-7.

17 Steptoe A, Breeze E, Banks J, et al. Cohort profile: the English longitudinal study of ageing. Int J Epidemiol 2013;42:1640-8.

18 Lee DM, Nazroo J, O'Connor DB, et al. Sexual health and well-being among older men and women in England: findings from the English Longitudinal Study of Ageing. Arch Sex Behav 2016;45:133-44.

19 DeSalvo KB, Bloser N, Reynolds K, et al. Mortality prediction with a single general self-rated health question. J Gen Intern Med 2006;21:267-75.

20 Steptoe A, Jackson SE. The life skills of older Americans: association with economic, psychological, social, and health outcomes. Sci Rep 2018;8:9669.

21 Banks J, Karlsen S, Oldfield Z, et al. Socio-economic position. In: Marmot A, ed. Health, wealth and lifestyle of the older population in England. London: Institute for Fiscal Studies, 2003.

22 Demakakos P, Hamer M, Stamatakis E, et al. Low-Intensity physical activity is associated with reduced risk of incident type 2 diabetes in older adults: evidence from the English Longitudinal Study of Ageing. Diabetologia 2010;53:1877-85.

23 Hamer M, Molloy GJ, de Oliveira C, et al. Leisure time physical activity, risk of depressive symptoms, and inflammatory mediators: the English Longitudinal Study of Ageing. Psychoneuroendocrinology 2009;34:1050-5.

24 Steffick DE. Documentation of affective functioning measures in the health and retirement study. HRS documentation report, 2000.

25 Anand P, Kunnumakara AB, Sundaram C, et al. Cancer is a preventable disease that requires major lifestyle changes. Pharm Res 2008;25:2097-116.
26 Chiuve SE, Rexrode KM, Spiegelman D, et al. Primary prevention of stroke by healthy lifestyle. Circulation 2008;118:947-54.

27 Franks P, Gold MR, Fiscella K. Sociodemographics, self-rated health, and mortality in the US. Soc Sci Med 2003;56:250514.

28 Jackson SE, Yang L, Veronese N, et al. Sociodemographic and behavioural correlates of lifetime number of sexual partners: findings from the English Longitudinal Study of Ageing. BMJ Sex Reprod Health 2019;45:138-46.

29 Mackenbach JP, Stirbu I, Roskam A-JR, et al. Socioeconomic inequalities in health in 22 European countries. N Engl J Med 2008;358:2468-81.

30 Poole L, Steptoe A. Depressive symptoms predict incident chronic disease burden 10 years later: findings from the English Longitudinal Study of Ageing (ELSA). J Psychosom Res 2018;113:30-6.

31 Robards J, Evandrou M, Falkingham J, et al. Marital status, health and mortality. Maturitas 2012;73:295-9.

32 Smith GD, Chaturvedi N, Harding S, et al. Ethnic inequalities in health: a review of UK epidemiological evidence. Crit Public Health 2000;10:375-408.

33 Van der Kooy K, van Hout H, Marwijk H, et al. Depression and the risk for cardiovascular diseases: systematic review and meta analysis. Int J Geriatr Psychiatry 2007;22:613-26.

34 Bridges S, Hussey D, Blake M. The dynamics of aging: the 2012 English Longitudinal Study of Ageing (Wave 6) - Technical Report. UK: NatCen Social Research, 2015.

35 Trienekens SCM, van den Broek IVF, Donker GA, et al. Consultations for sexually transmitted infections in the general practice in the Netherlands: an opportunity to improve STI/ HIV testing. BMJ Open 2013;3:e003687.

36 Agudo A, Bonet C, Travier N, et al. Impact of cigarette smoking on cancer risk in the European prospective investigation into cancer and nutrition study. J Clin Oncol 2012;30:4550-7.

37 Bagnardi V, Blangiardo M, Vecchia CL, et al. A meta-analysis of alcohol drinking and cancer risk. Br J Cancer 2001;85:1700-5.

38 Bergmann MM, Byers T, Freedman DS, et al. Validity of selfreported diagnoses leading to hospitalization: a comparison of self-reports with hospital records in a prospective study of American adults. Am J Epidemiol 1998;147:969-77.

39 Bush TL, Miller SR, Golden AL, et al. Self-report and medical record report agreement of selected medical conditions in the elderly. Am J Public Health 1989;79:15546.

40 Simpson CF, Boyd CM, Carlson MC, et al. Agreement between self-report of disease diagnoses and medical record validation in disabled older women: factors that modify agreement. J Am Geriatr Soc 2004;52:123-7. 